\title{
A GLIMPSE AT KNOT THEORY
}

\author{
PAWEł TRACZYK \\ Instytut Matematyki, Uniwersytet Warszawski \\ Banacha 2, 02-097 Warszawa \\ E-mail: traczyk@mimuw.edu.pl
}

The purpose of this essay is two-fold. We want to give the reader some elementary idea of what knot theory is about and to provide some information of the recent progress in the area, especially in its combinatorial branch. This is meant as an elementary introduction for non-specialists with an interest in the area.

We would like to get straight to the point, so we omit the discussion of all the technicalities. Generally, knot theory deals with one-dimensional closed piecewise linear submanifolds of the three-dimensional sphere $S^{3}$, in other words we consider the images of piecewise linear embeddings of disjoint unions of circles into $S^{3}$. These are called links, or in the case of just one component-knots. In the case of a single component this is our mathematical model of a knotted closed string in the physical space. Mathematically, it is important that the knot be closed, because from the topologist's point of view the embeddings of a segment into $S^{3}$ are all equivalent. It is surprising, how the natural idea of equivalence of knots (or links) proves difficult to be expressed mathematically. It is commonly agreed that the most appropriate mathematical description of this equivalence is by means of ambient isotopy. This means that two links $L_{1}$ and $L_{2}$ are equivalent if there exist a one-parameter continuous family $H$ of homeomorphisms of $S^{3}$ into itself such that $H_{1}=$ identity and $H_{2}\left(L_{1}\right)=L_{2}$. It would be more natural to consider a one parameter family of embeddings starting with $L_{1}$ and ending with $L_{2}$ - this seems closer to the intuitive idea of deforming the links without cutting them, but this leads to nowhere - under such definition all knots would be equivalent.

Our subject in this essay is the combinatorial knot theory. This means that we practically forget the whole three-dimensional context of the situation and

1991 Mathematics Subject Classification: Primary: 57M25.

Lecture given at the Banach Center Colloquium on 18th March 1993.

The paper is in final form and no version of it will be published elsewhere. 
do all our work with knot diagrams on a plane or better on a two-dimensional sphere. What is meant by knot (or link) diagram is a certain very natural form of visualisation of the subject: we consider regular projections of links onto a plane (regular means that we choose the projection angle to avoid multiple points as much as possible - the only multiple points that we cannot avoid are double points which we call crossings) with an indication of which arc is over and which is under at every crossing. Figure 1 below shows an example of such a diagram.

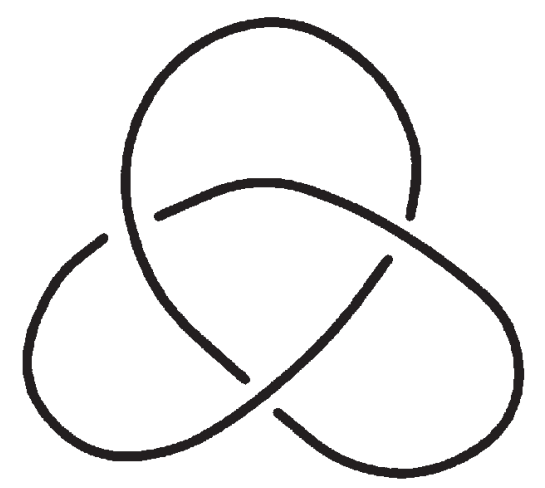

Figure 1

The knot shown in this figure is the so-called trefoil. It is not equivalent to the trivial knot, which is intuitively obvious but not so easy to prove. There is a fundamental theorem showing how the three-dimensional problem of equivalence of links is reduced to this two-dimensional setting.
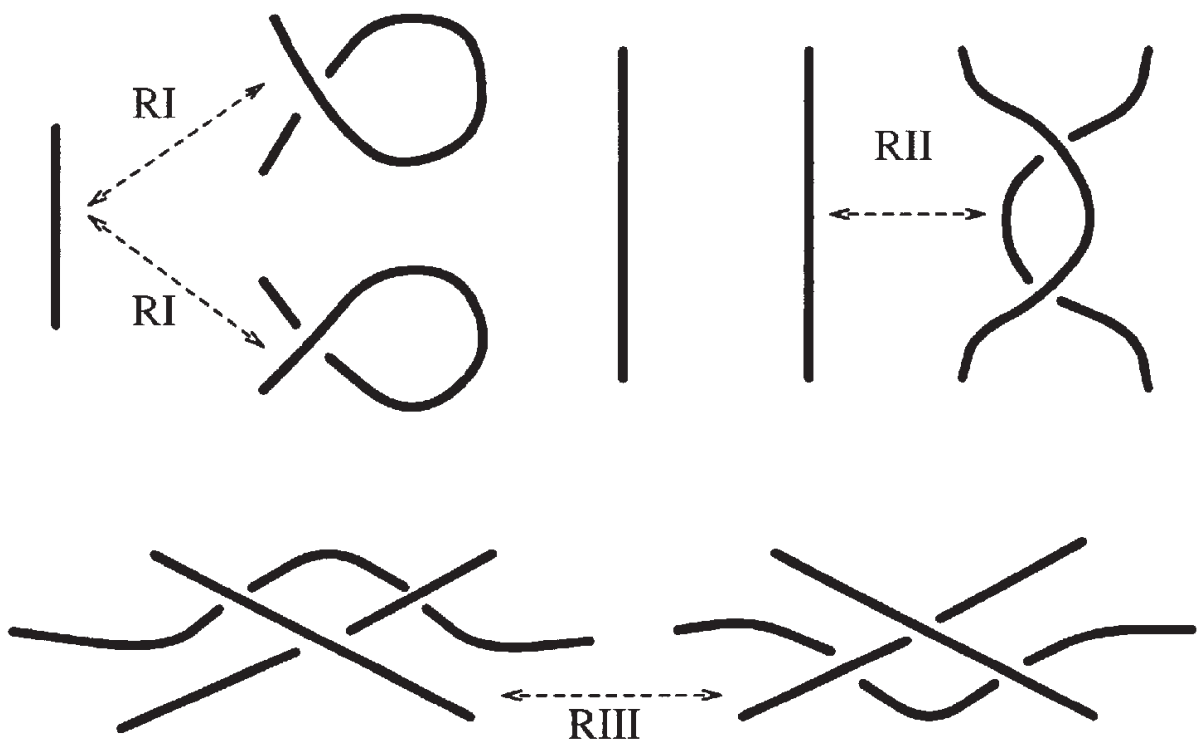

Figure 2 
THEOREM (Reidemeister). Two diagrams represent equivalent links if and only if one may be transformed into the other by a sequence of operations given in Figure 2 (the so-called Reidemeister moves of type I, II and III).

The most obvious problem in knot theory is that of classification of links. The natural way to address this problem is to construct invariants of links, that is such functions that ascribe some mathematical quantities to links in a way that depends only on the equivalence class of the given link. One classical example is the fundamental group of the complement of the given link in $S^{3}$. This invariant has the advantage of being quite obviously invariant. It is also quite easy to write down a finite presentation of the considered group (given the diagram), which is called Wirtinger presentation (see [B-Z], chapter 3). Another advantage is that this invariant is quite effective in distinguishing knots. In fact, the fundamental group with certain additional refinement - the so-called peripheral system classifies knots. But there is one great disadvantage: although theoretically very nice, the invariant is very difficult to use in practice. This is so because the isomorphism problem for group presentations is very difficult.

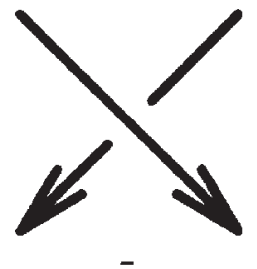

$-$

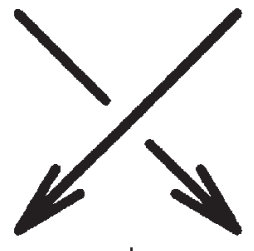

$+$

Figure 3

We want to present here a class of combinatorial invariants discovered since 1984, known as knot polynomials. What we mean by combinatorial is that the invariants are defined through various types of diagrams and their invariance is proved by means of Reidemeister moves. It is very educational to have a look first at one of them, denoted by $f$. This is the Kauffman version of the Jones polynomial $V$ (the polynomial $V$ was introduced by V.F.R. Jones in 1984 in a very influential paper $[\mathrm{J}]$ ). The polynomial $f$ is a polynomial in two variables $A$ and $A^{-1}$ with integer coefficients. It is defined for oriented links. The definitions is made in two stages. In the first stage we forget about the orientation and compute the Kauffman bracket $\langle D\rangle$ of the unoriented version of the given diagram $D$. The polynomial $f_{D}$ of the original oriented diagram is then defined by simply multiplying the bracket of $D$ by $\left(-A^{3}\right)^{-w(K)}$, where $w(K)$ is the sum of the signs of crossings as defined in Figure 3.

Now, what is the bracket of an unoriented diagram $D$ ? This is defined in a very simple recursive way.

1. $\langle\bigcirc\rangle=1$ (here $\bigcirc$ is simply the trivial diagram).

2. $\langle D \sqcup \bigcirc\rangle=\left(-A^{-2}-A^{2}\right)\langle D\rangle$ (here, $D \sqcup \bigcirc$ means $D$ with an additional trivial circle added to it). 
3. see Figure 4 below.

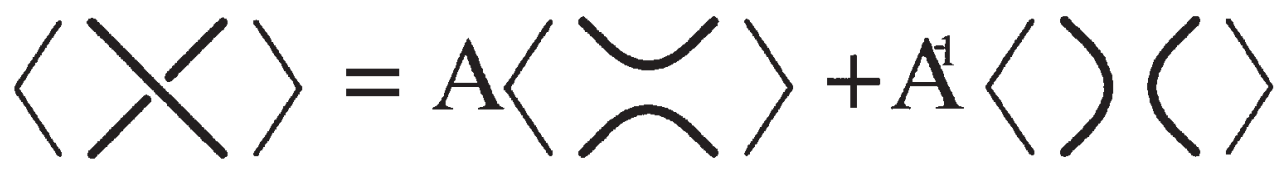

Figure 4

Without some explanation Figure 4 is a little cryptic. In fact it introduces one of the most important ideas that were discovered in knot theory in the recent years. What is considered in Figure 4 is a triple of almost identical diagrams. They differ only locally and what the figure shows (inside of the three brackets) is this tiny area in which the diagrams do differ - one diagram has a crossing in this area, in the remaining two the crossing is replaced (smoothed) in two different ways.

If the choice of coefficients in the above formulas seems a little arbitrary, the reader is advised to carry out the calculations proving that the bracket is invariant under Reidemeister moves of types II and III, then to check how it changes when a Reidemeister I move is performed. It is surprising that such a simple definition leads to a very powerful invariant of links. As was mentioned above, the polynomial $f$ is a version of the polynomial $V$ originally introduced by Jones. This was introduced by means of representations of braid groups into certain von Neumann algebras, but was soon discovered to satisfy the following equality:

4. $t^{-1} V_{+}-t V_{-}=\left(\sqrt{t}-\frac{1}{\sqrt{t}}\right) V_{0}$,

where $V_{+}, V_{-}$and $V_{0}$ are again the polynomials of three diagrams that are almost identical and differ only locally as shown in Figure 5.
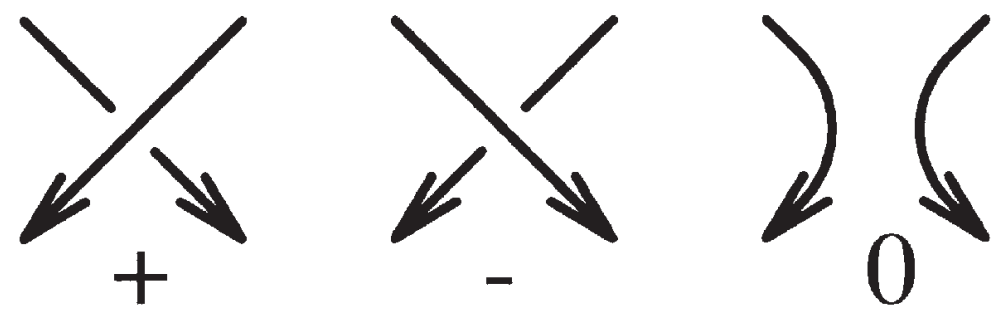

Figure 5

Let us stress the difference: this time we consider oriented diagrams, this is why we consider two versions of the crossing and just one version with the crossing removed (because only this version may be coherently oriented). Historically, it is this equality that started the revolution in knot theory. It was soon discovered that in this case the choice of coefficients does not really matter - one can replace $t^{-1}$, $-t$ and $\sqrt{t}-\frac{1}{\sqrt{t}}$ with practically anything, and still get an invariant satisfying the relevant condition. While the most obvious choice of 'practically anything' would 
probably be three independent (but commuting) variables $x, y$ and $z$, it turns out that a wiser choice is the one used in the formula below:

5. $l P_{+}+l^{-1} P_{-}+m P_{0}=0$.
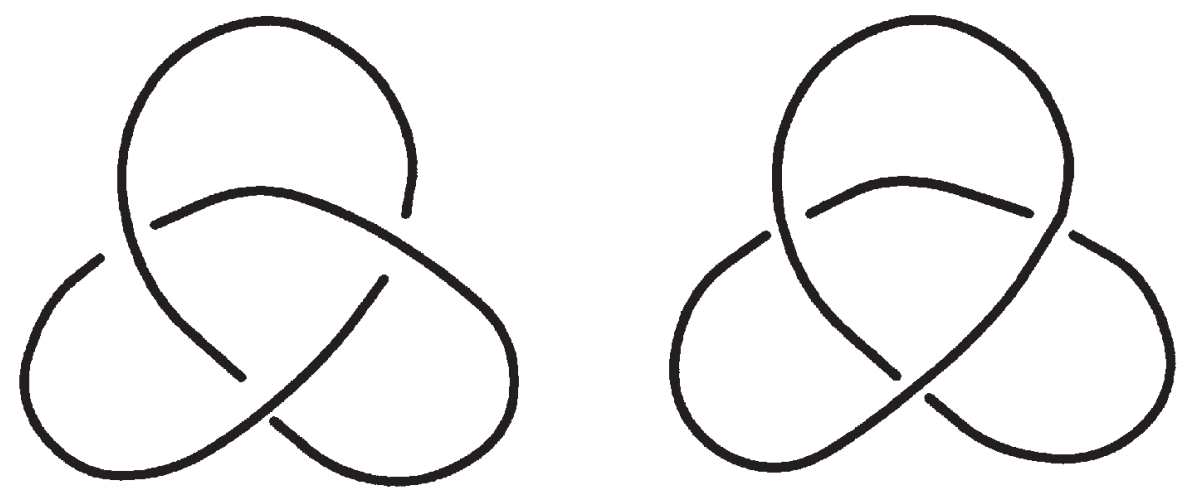

Figure 6

Surprisingly, no information is lost (compared to the $x, y$ and $z$ version) - the two invariants are fully equivalent. The invariant $P$ defined by equality 5 and the condition $P(\bigcirc)=1$ is known as the Homfly polynomial. It was discovered at more or less the same time by five groups of researchers (see [FYHLMO]). Since then hundreds of papers have been written in the area, exploring various properties of the new invariants and introducing new invariants of similar construction. Of these, the most significant one was the Kauffman polynomial (see $[\mathrm{K}]$ ). Some important theoretical results were proved by means of the Jones polynomial, especially two of the so-called Tait's conjectures. These are conjectures dating back to the nineteenth century. Let us describe one of them. We consider alternating diagrams. These are diagrams in which undercrossings and overcrossings are encountered alternatingly when one walks along the diagram. Thus in Figure 6 the diagram on the left is alternating and the diagram on the right is not.

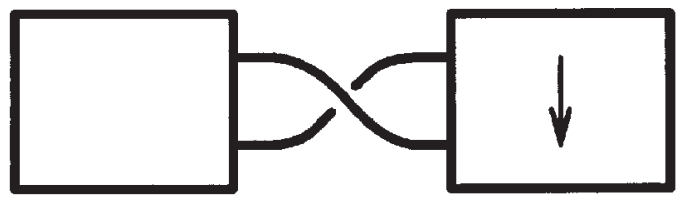

Figure 7

In some cases an alternating diagram may look as in Figure 7.

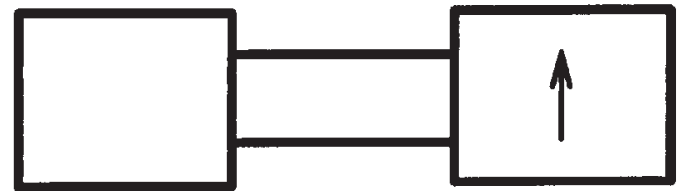

Figure 8 
It is then possible to modify it to the form given in Figure 8 and the result is still an alternating diagram.

When this is no longer possible we say that the diagram is reduced. Now, the first Tait conjecture says that an alternating reduced diagram has the minimal possible number of crossings (for the given class of equivalence of knots). It turns out that the span of the Jones polynomial (the difference between the highest and the lowest exponents) is always smaller than or equal to the number of crossings of the considered diagram, and in the case of reduced alternating diagrams the equality always holds. This proves the conjecture in a surprisingly elegant way. For the details see $[\mathrm{M}],[\mathrm{K}]$ and $[\mathrm{T}]$. The last paper is especially interesting because it explores another aspect of the story - the connections of knot theory and the graph theory.

Generally speaking the astounding career of the new invariants is to a large degree due to deep and unexpected connections with various aspects of mathematics and physics that were discovered in the aftermath of the introduction of the Jones polynomial. To the reader with an interest in this fascinating story the author wants to recommend the book [K1].

\section{References}

[FYHLMO] P. Freyd, D. Yetter, J. Hoste, W. B. R. Lickorish, K. Millett, A. Ocneanu, A new polynomial invariant of knots and links, Bull. Amer. Math. Soc. 12 (1985) 239-249.

[J] V. F. R. Jones, A polynomial invariant for knots via a von Neumann algebras, Bull. Amer. Math. Soc. 12 (1985) 103-111.

[K] L. Kauffman, State models and the Jones polynomial, Topology 26 (1987) 395407.

[K1] L. Kauffman, Knots and Physics, World Scientific, Singapore-New JerseyLondon-Hong Kong (1991).

[M] K. Murasugi, Jones' polynomial and classical conjectures in knot theory, Topology 26 (1987), 187-194.

[T] M. B. Thistlethwaite, A spanning tree expansion of the Jones polynomial, Topology 26 (1987), 297-309. 